\title{
IDENTIFICATION OF PARAMETRIC MODELS WITH A PRIORI KNOWLEDGE OF PROCESS PROPERTIES
}

\author{
KRZYSZTOF B. JANISZOWSKI ${ }^{a, *}$, PAWEŁ WNUK ${ }^{a}$ \\ ${ }^{a}$ Institute of Automatic Control and Robotics \\ Warsaw University of Technology, Św. A. Boboli 8, 02-525 Warsaw, Poland \\ e-mail: kjanisz@mchtr.pw.edu.pl
}

\begin{abstract}
An approach to estimation of a parametric discrete-time model of a process in the case of some a priori knowledge of the investigated process properties is presented. The knowledge of plant properties is introduced in the form of linear bounds, which can be determined for the coefficient vector of the parametric model studied. The approach yields special biased estimation of model coefficients that preserves demanded properties. A formula for estimation of the model coefficients is derived and combined with a recursive scheme determined for minimization of the sum of absolute model errors. The estimation problem of a model with known static gains of inputs is discussed and proper formulas are derived. This approach can overcome the non-identifiability problem which has been observed during estimation based on measurements recorded in industrial closed-loop control systems. The application of the proposed approach to estimation of a model for an industrial plant (a water injector into the steam flow in a power plant) is presented and discussed.
\end{abstract}

Keywords: absolute error measure, constrained parameters estimation, identification, parametric MISO models.

\section{Introduction}

Identification focused on determination of a statistic model of the process, which would represent dynamic properties of a plant, is nowadays one of the basic approaches to the development of advanced modern control systems. The model can be developed in various structures, both linear and non-linear. The only basic demand is its quality and fidelity in representing the given process properties. Verification of model behavior is usually based on comparison of model output $y_{m}(t)$ with process output $y(t)$, which should be close in a defined norm.

The output measurements $y(t)$ are recorded in the presence of measured (or estimated) inputs $v_{i}(t)$, which can express controls $u_{i}(t)$ or disturbances $w_{i}(t)$. In industrial applications many signals are usually measured-this amount can reveal information about process behavior, however, not all should be included into the statistic model structure, so as not to mask real impact of other inputs. For example, consider a chemical reactor, where the reaction intensity $I$ of a mixture in the reactor is dependent on temperature $T$

* Corresponding author of this mixture. The impact of the heating steam flow $H$, being one of the control inputs, is very important in modeling the reaction $I$, but introduction of mixture temperature $T$ into the set of statistic model inputs can conceal the effect induced by control $H$, and as a result, the estimated model may represent a completely false reaction of intensity $I$ to variations in $H$. On the other hand, the model of intensity $I$ without temperature $T$ will not involve information on unmeasurable disturbances which may influence intensity $I$, like, e.g., changed heat transfer terms, a neglected saturation of the steam, its flow or pressure, and many other factors. Another important aspect is a proper excitation by manipulated controls in data used for identification of the process model. A well-known effect is the loss of identifiability (Astrom, 1983; Eykhoff, 1974; Heath, 2001; Isermann, 1988; Ljung, 1999; Ljung and Foorsell, 1998; Norton, 1980; Söderström and Stoica, 1989) of a model based on direct measurements of controller input $u$ and output $y$ from the process, if the investigated process runs in a closed-loop system without additional control excitation or demanded changes in the value of $y_{d}(t)$.

In problems where representative signals are measured, the most efficient way is modeling the 
investigated system based on a description of the system with known relations and values of model coefficients $\Theta$ fitted to recorded measurements (e.g., Gautier and Briot, 2011). Although this way is very effective and robust, it is useful for processes with well-developed models and low impact of unmeasured disturbances.

Usually, during process identification, some a priori information of the investigated process is accessible due to the process operator's knowledge, balance of the flows or energy exchange, but it is limited to very basic information like, e.g., approximated static gains for basic controls, main inertia or transportation delays. Although this information is accessible, there is no direct way to introduce it into the estimated statistic model structure. Many authors, who are focused on statistical applications (Bun and Carree, 2000; Hayakawa, 2010; Kiviet, 1995; Phillips and Sul, 2007), try to reduce the bias effects introducing this information in a different way into simple, first order signal statistics. Others (e.g., Ferretti et al., 1991; Gourieroux et al., 2010; Hayakawa, 2010) test various approaches and methods with respect to their sensitivity to biases.

Aguire et al. (2004) presented an application of LS estimation to a problem of identification of non-linear characteristic coefficients in a case of some known constraints. There are also methods based on identification of models of plant dynamics, e.g., in a continuous time domain (Heath, 2001; Kowalczuk and Kozłowski, 2000; Söderström et al., 1997), or in forms different from the traditional discrete-time transfer function representation (e.g., Goodwin and Welsh, 2002; Ninness et al., 1999). In the area of control applications the main problems arise in the case of estimation of plant dynamics, based on data recorded for a plant $P$ working in a control loop system with a controller $C$. In the case of the closed control loop operating at a steady working point without additional excitations, the estimation leads to determination of the plant dynamics as $-1 / C$, which is called the loss of identifiability (Astrom, 1983; Goodwin and Welsh, 2002; Ljung, 1999; Ljung and Gunnarson, 1990; Norton, 1980; Söderström and Stoica, 1989). This effect was observed and some remedies were proposed (e.g., Goodwin and Welsh, 2002), but they in fact limit the possibility of model identification by reduction of its order or the transfer domain. On the other hand, the above-mentioned effect of the loss of identifiability was not reported in the investigation of models in continuous time domain (e.g., Kowalczuk and Kozłowski, 2000; Ljung and Foorsell, 1998; Söderström et al., 1997), hence perhaps this approach can be more robust to deal with the above-mentioned effect.

The paper is organized as follows. After the introductory section, the problem statement together with a short discussion of possible applications is described in Section 2. Section 3 contains derivation of constrained estimation, based on known a priori information, with application of a special weighted least-squares approach. This approach can be transformed to estimation in terms of the performance index being the sum of absolute errors and the corresponding on-line algorithm is presented in detail in Section 4. The major advantage of this version of the algorithm is its low sensitivity to measurement outliers, which can be significant in the case of small variations in the measured signals (Janiszowski, 2014). In Section 5 an application of this algorithm to estimation of the model of a water injector into the steam flow in a power plant, operating in a closed-loop system, is presented. After a detailed discussion of the estimation results, where the effect of weak identifiability is observed, the paper is finished with concluding remarks in Section 6.

\section{Problem statement}

Consider a process with measured controls $u_{i}$, disturbances $w_{j}$ and process output signal $y$. We are looking for a linear (with respect of coefficients) discrete-time model $y_{m}$ of the process output $y$, expressed by the relation

$$
y_{m}(k)=v(k) \Theta, \quad v, \Theta \in \mathbb{R}^{m},
$$

where $v(k)=\left[v_{1}(k), \ldots, v_{m}(k)\right]$ is a row vector of signals $v_{i}(k)$ dependent on measured or estimated signals $u_{i}, w_{j}$ or $y$. The row vector $v(k)$ is further called the vector of model inputs and $\Theta$ is a column vector of unknown model coefficients, further called the model. The investigated process is assumed to be stationary in the sense that estimation of process dynamics in the form (1) is justified for the given operation point of the investigated process.

The employed estimation algorithms of $\Theta$ are usually derived with application of some standard formulation of the estimation problem, e.g., the least sum of square errors of model output or the expected value of the variance for the one-step prediction error of the model (Eykhoff, 1974; Isermann, 1988; Ljung, 1999; Norton, 1980; Söderström and Stoica, 1989). Let us denote by $I_{M}$ the performance index used by the estimation method $M$ for determination of the model $\Theta$ and based on minimization of a measure $\|\cdot\|$ of the model error $e=y-y_{m}$ as

$$
I_{M}(\hat{\Theta})=\min _{\Theta}\|e\|
$$

where $\hat{\Theta}$ is the optimal solution in terms of the index $I_{M}$. The estimated model $\hat{\Theta}$ can then be verified by determination of the value of the application index $I_{D}$, which expresses the intended destination of the model $\hat{\Theta}$,

$$
I_{D}(\hat{\Theta})=F(\hat{\Theta}),
$$


defined as a functional $F$ of the vector $\hat{\Theta}$ representing the final application of the estimated model $\hat{\Theta}$. For example, this functional can represent a quality measure of the modeling error in the control system considered regarding recorded disturbances,

$$
F(\hat{\Theta})=\sum_{k=p}^{L}\left|y_{D}(k)-\hat{v}_{D}(k) \hat{\Theta}\right|,
$$

where the signal $y_{D}$ represents data recorded in some experiment $D$ used for verification of the quality of model $\hat{\Theta}$. The vector $\hat{v}_{D}(k)$ of model inputs preserves the same structure as $v(k)$, being combined from measured signals $u_{D_{i}}(k), w_{D_{j}}(k)$ but with values corresponding to the output, estimated by the model output $y_{m}(k)$ (1) instead of the measured values $y(k)$. The number $N=$ $L-p+1$ of the measurements (4), taken into account, depends on all the measurement instants $L$ and the initial discrete time instant $p$. The measure $F$ in the form (4) cannot be used directly as the index for optimization of the statistic estimator (2), but can be used for evaluation of the applicability of the estimated model $\hat{\Theta}$.

In the formulated problem there is a possibility of testing different model structures and approaches (linear, non-linear, neural nets of various structures, etc.) used in its calculation, and then the model $\hat{\Theta}$, which represents the least value of the application index (4), can be chosen.

The problem of identification, i.e., determination of the model $\hat{\Theta}$ which yields the least value of $I_{D}$, will be now extended-the estimated model should satisfy additional conditions which express a priori knowledge of the plant, i.e., gains of step responses, inertia, or influence of mutual compensating inputs. This a priori knowledge of process properties will be introduced in the form of a linear relation between the components of the coefficient vector $\hat{\Theta}$,

$$
Q \hat{\Theta}=q, \quad q \in \mathbb{R}^{p}, \quad Q \in \mathbb{R}^{p \times m},
$$

where the product $Q Q^{T}$ is nonsingular. The condition formulated as the relation (5) expresses a priori information on the estimated process. This information can introduce different properties, but its linear form (5) is crucial. Examples of the formula (5) are discussed in Section 3.

\section{Problem solution}

Let us consider an estimation method $M$ of the model coefficients vector $\hat{\Theta}$ based on the model error

$$
\begin{gathered}
\varepsilon=Y-V \Theta, \quad \varepsilon, Y \in \mathbb{R}^{N}, \quad V \in \mathbb{R}^{N \times m}, \\
Y=\left[\begin{array}{c}
y(p) \\
\vdots \\
y(M)
\end{array}\right], \quad V=\left[\begin{array}{c}
\nu(p) \\
\vdots \\
\nu(M)
\end{array}\right], \quad \varepsilon=\left[\begin{array}{c}
\varepsilon(p) \\
\vdots \\
\varepsilon(M)
\end{array}\right],
\end{gathered}
$$

and a minimized measure of the model error, defined as

$$
I_{M}(\Theta)=\varepsilon^{T} W \varepsilon,
$$

where $W$ is a positive-definite weight matrix. The estimation of the optimal vector coefficients vector $\Theta^{O}$ in terms of the minimum of the index (7) will be defined by the well-known formula (Eykhoff, 1974; Isermann, 1988; Ljung, 1999; Norton, 1980; Söderström and Stoica, 1989)

$$
\Theta^{0}=\left[V^{T} W V\right]^{-1} V^{T} W Y, \quad \Theta^{0} \in \mathbb{R}^{m} .
$$

The investigated estimation problem will involve a bias by introducing a bound defined by (5), i.e., the solution to the estimation problem with the bound (5) will minimize the index in the form of Eqn. (7).

Theorem 1. The vector $\Theta$ of the coefficients of the model (1), which satisfies the condition (5) and minimizes the error measure $I_{M}$, has the form

$$
\Theta^{*}=\Theta^{0}-\zeta\left[Q \Theta^{0}-q\right], \quad \Theta^{*} \in \mathbb{R}^{m},
$$

where

$$
\begin{gathered}
\zeta=S Q^{T}\left[Q S Q^{T}\right]^{-1}, \\
S=\left[V^{T} W V\right]^{-1} .
\end{gathered}
$$

Proof. Estimation of the model vector $\Theta$, which minimizes (7) subject to bounds (5), can be considered as minimization of the performance index in the form

$$
\begin{aligned}
I^{*}(\Theta, \zeta)= & {[Y-V \Theta]^{T} W[Y-V \Theta] } \\
& +\zeta(Q \Theta-q), \quad \zeta \in \mathbb{R}^{p},
\end{aligned}
$$

where $\zeta$ is the vector of Lagrange multipliers. From the necessary conditions for the model vector $\Theta^{*}$ to be a minimum of $I^{*}$, we obtain

$$
\begin{gathered}
\frac{\partial}{\partial \Theta} I^{*}(\Theta, \zeta)=-2 V^{T} W Y+2 V^{T} W V \Theta^{*} \\
+Q^{T} \zeta^{*}=0 \\
\frac{\partial}{\partial \zeta} I^{*}(\Theta, \zeta)=Q \Theta^{*}-q=0
\end{gathered}
$$

which can be expressed as a set of linear equations,

$$
\begin{aligned}
& {\left[\begin{array}{cc}
\alpha & Q^{T} \\
Q & 0
\end{array}\right]\left[\begin{array}{l}
\Theta^{*} \\
\zeta^{*}
\end{array}\right]=\left[\begin{array}{l}
\beta \\
q
\end{array}\right],} \\
& \alpha=2 V^{T} W V, \quad \beta=2 V^{T} W Y,
\end{aligned}
$$

with a solution for $\Theta^{*}$ being a product of the upper row of inversion of the block matrix

$$
A=\left[\begin{array}{cc}
\alpha & Q^{T} \\
Q & 0
\end{array}\right]
$$


and the vector

$$
\left[\begin{array}{l}
\beta \\
q
\end{array}\right]
$$

When the inverted block matrix

$$
A=\left[\begin{array}{ll}
A_{1} & A_{2} \\
A_{3} & A_{4}
\end{array}\right]
$$

has a non singular submatrix $A_{1}$ (equal to $\alpha$ in (13)), the inversion of $A$ can be expressed in the form

$$
A^{-1}=\left[\begin{array}{cc}
A_{1}^{-1}+A_{1}^{-1} A_{2} \Lambda^{-1} A_{3} A_{1}^{-1} & A_{1}^{-1} A_{2} \Lambda^{-1} \\
-\Lambda^{-1} A_{3} A_{1}^{-1} & \Lambda^{-1}
\end{array}\right]
$$

with

$$
\Lambda=A_{4}-A_{3} A_{1}^{-1} A_{2} .
$$

In the case considered $A_{4}=0$ and we have $\Lambda=$ $-Q \alpha^{-1} Q^{T}$. The upper row of the inverted block matrix $A^{-1}$ is equal to $\left[\alpha^{-1}+\alpha^{-1} Q^{T} \Lambda^{-1} Q \alpha^{-1}, \alpha^{-1} Q^{T} \Lambda^{-1}\right]$, and as a result we can determine the investigated vector of the model as

$$
\Theta^{*}=\left(\alpha^{-1}+\alpha^{-1} Q^{T} \Lambda^{-1} Q \alpha^{-1}\right) \beta-\alpha^{-1} Q^{T} \Lambda^{-1} q .
$$

Now we can observe that the product $\alpha^{-1} \beta$ is equal to $\Theta^{0}$ defined in (8), and the formula for $\Theta^{*}$ can be rearranged as

$$
\begin{aligned}
\Theta^{*} & =\alpha^{-1} \beta+\alpha^{-1} Q^{T} \Lambda^{-1}\left[Q \alpha^{-1} \beta-q\right] \\
& =\Theta^{0}+\alpha^{-1} Q^{T} \Lambda^{-1}\left[Q \Theta^{0}-q\right],
\end{aligned}
$$

which is equal to the formula presented in (8).

The above formula (3) is similar to the result presented by Draper and Smith (1998) for the case of a constrained least-squares estimation problem.

The biased estimate $\Theta^{*}$ proposed in (9) will converge to $\Theta^{0}$ when the model $\Theta^{0}$ preserves the property defined by the condition (5). Then the bias term $\alpha^{-1} Q^{T} \Lambda^{-1}\left[Q \Theta^{0}-q\right]$ will vanish from the estimation $(9]$. Now, let us consider possible application cases where the biased (9) formula can be applied. First, the problem of a simple, linear, dynamic SISO system will be investigated. A model of the discrete-time transfer function for this system with a sampling interval $\Delta$ and the $\mathrm{ZOH}$ unit is represented by the transfer function

$$
\begin{aligned}
G\left(z^{-1}\right) & =\frac{Y\left(z^{-1}\right)}{U\left(z^{-1}\right)}=\frac{B\left(z^{-1}\right) z^{-d}}{A\left(z^{-1}\right)} \\
& =\frac{b_{0} z^{-d}+b_{1} z^{-1-d}+\cdots+b_{r} z^{-r-d}}{1+a_{1} z^{-1}+\cdots+a_{r} z^{-r}}
\end{aligned}
$$

where $z^{-1}$ is a shift operator in the discrete time domain, $z^{-s} v(k)=v(k-s), d$ is a discrete form of the delay $\tau=d \Delta$. The transfer function (18) can be transformed into a difference equation for the output $y(k)$. The relation between the input and output values, assuming that $y(k)$ is the effect of the dynamics only and the previous values of input $u(k-i)$, is equal to

$$
y(k)=-\sum_{i=1}^{r} a_{i} y(k-i)+\sum_{i=0}^{r} b_{i} u(k-i-d) .
$$

The model of these dynamics expressed in the form (1) is simple,

$$
\begin{aligned}
y(k)= & v(k) \Theta, \\
v(k)= & {[-y(k-1), \ldots,-y(k-r),} \\
& u(k-d), \ldots, u(k-r-d)], \\
\Theta= & {\left[a_{1}, \ldots, a_{r}, b_{0}, \ldots, b_{r}\right]^{T} . }
\end{aligned}
$$

Let us now suppose that a priori information on this system is the static gain $K$ of the above transfer function (18). Then, from the definition of (18) we can write

$$
\begin{aligned}
K=\frac{b_{0}+b_{1}+\cdots+b_{r}}{1+a_{1}+\cdots+a_{r}} & \Rightarrow \\
K\left(1+a_{1}+\cdots+a_{r}\right) & =\left(b_{0}+b_{1}+\cdots+b_{r}\right)
\end{aligned}
$$

and express the above condition in the form (5) as

$$
\begin{gathered}
{[-1, \ldots,-1,1 / K, \ldots, 1 / K] \Theta=Q \Theta=q=1} \\
Q=[-1, \ldots,-1,1 / K, \ldots, 1 / K]
\end{gathered}
$$

In the case of many inputs into the process (the case of a MISO system) and the knowledge of some of static gains $K_{i}$, one can derive the model

$$
\begin{aligned}
y(k)= & v(k) \Theta, \quad v(k), \Theta \in \mathbb{R}^{M}, \\
M= & r+m(r+1), \\
v(k)= & {[-y(k-1), \ldots,-y(k-r),} \\
& \left.u_{1}\left(k-d_{1}\right), \ldots, u_{m}\left(k-r-d_{m}\right)\right], \\
\Theta= & {\left[a_{1}, \ldots, a_{r}, b_{10}, \ldots, b_{1 r}, \ldots, b_{m 0}, \ldots, b_{m r}\right]^{T} . }
\end{aligned}
$$

If there are known static gains, e.g., $K_{1}$ and $K_{3}$ for the first and third inputs, the linear form of the bounds (5) will be defined by $Q$,

$$
\begin{aligned}
Q \Theta & =q, \quad v(k), q \in \mathbb{R}^{2}, \quad Q \in \mathbb{R}^{2 \times M}, \\
q & =[1,1]^{T}, \\
Q & =\left[\begin{array}{l}
-1, \ldots,-1, \frac{1}{K_{1}}, \ldots, \frac{1}{K_{1}}, 0, \ldots, 0,0, \ldots, 0 \\
-1, \ldots,-1,0, \ldots, 0, \frac{1}{K_{3}}, \ldots, \frac{1}{K_{3}}, 0, \ldots, 0
\end{array}\right] .
\end{aligned}
$$

It should be noted that the above conditions are specified without any restrictions on the model order. If additional precise information about the model coefficient 
vector is accessible, then it could be checked whether it can be included in the form of bounds expressed as in (5).

Another problem that can be solved by the proposed approach and existing in industrial applications of identification techniques arises in processes with integral action, i.e., the process output is in fact an integral of input activity. Identification based on recorded measurements is difficult, due to a variable output level, an undefined operation point and effects of the controller activity system, which hold the process output on a predefined level. The resulting estimation may contain the integration property, which usually is represented as inertia of a very large time constant. This a priori information can be included into estimation in the form (5), too. It is clear that the integrating action of the process described in the form of the transfer function (18), both for a single- and a multi-input case, will induce at least one unit root in the denominator of (18) and is in fact equal to the bound (5) in the form

$$
1+\sum_{i} a_{i}=0 \Rightarrow[1, \ldots, 1,0, \ldots, 0] \Theta=-1 .
$$

\section{Estimation algorithm}

The algorithm represented by the estimator (8) can express different approaches. In the case of the weights $W$ in (7) being equal, we obtain a simple least-squares approach. In the case of the weights determined on the basis of the covariance matrix of the model output residuals, one can obtain a form close to the filtered least-squares approach. When the weights are, e.g., fitted to each step of the algorithm, we can obtain a performance index close to the least sum of absolute model errors (LAE) (Janiszowski, 1998; 2014). This form of estimation was used because it is robust to local outliers in the processed data. This algorithm will be briefly recalled, for exposing terms necessary for determination of the estimate (9) and the products (10), (11). The presented on-line version can be used iteratively with the performance index (4) continually decreasing and converging to the global minimum of the LAE (for more details, see Kozłowski and Kowalczuk, 2007).

Algorithm 1 can be easily transformed to a simple least-squares approach by setting parameters $\lambda=0$ and $\gamma=2$.

The transformation of $\Theta^{0}$ into $\Theta^{*}$, made in Step 6 of Algorithm 1 is not necessary in each step, hence it is calculated only when needed. It requires inversion of the matrix and can be time consuming. The choice of the threshold value $\varepsilon_{\min }$ in Step 2 of the above algorithm also remains to be defined. Most authors suggest to use a value being a low ( 2 up to 5) multiplicity of measurement transducer quantization.

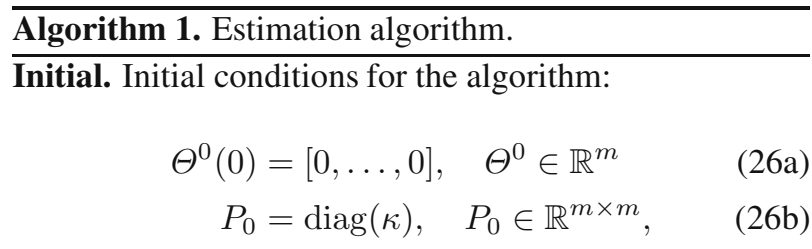

and $\kappa \in\left[10^{3}, 10^{5}\right]$ initializes the matrix $P$.

Step 1. Estimate the model error:

$$
\begin{aligned}
\varepsilon_{k} & =y(k)-v(k) \Theta^{0}(k-1), \quad k=1, \ldots, L, \\
v(k) & =\left[v_{1}(k), v_{2}(k), \ldots, v_{m}(k)\right] .
\end{aligned}
$$

Step 2. Determine the weight coefficient:

$$
\begin{aligned}
e_{k} & =\left\{\begin{array}{cl}
1 /\left|\varepsilon_{k}\right|^{\lambda}, & \left|\varepsilon_{k}\right| \geq \varepsilon_{\min }, \\
\max =1 / \varepsilon_{\min }, & \left|\varepsilon_{k}\right|<\varepsilon_{\min },
\end{array}\right. \\
\lambda & =2-\gamma,
\end{aligned}
$$

where $\gamma \in[1,2]$ is the exponent of the weights $e_{k}$ (28) equal to 2 in the case of the sum of the squared deviations or 1 in the case of the sum of absolute errors (Janiszowski, 2014).

Step 3. Calculate the vector of the weighted model inputs $w(k)$ and the vector $\chi$ :

$$
\begin{aligned}
w(k) & =e_{k} v(k), \\
\mu(k) & =\left[\rho+w(k) P_{k+1} w(k)^{T}\right]^{-1}, \\
\chi & =\mu(k) P_{k-1} w(k)^{T},
\end{aligned}
$$

where $\rho \in[0.99,1]$ is a forgetting factor and $P_{k-1}$ is a matrix approximating $\left[V^{T} W V\right]^{-1}$ at each step of the on-line identification algorithm.

Step 4. Update the model coefficient vector:

$$
\Theta^{0}(k)=\Theta^{0}(k-1)+\operatorname{sign}\left(\varepsilon_{k}\right) \chi\left|\varepsilon_{k}\right|^{\gamma-1} .
$$

where the term $\operatorname{sign}\left(\varepsilon_{k}\right)$ expresses the ratio $\varepsilon_{k} / e_{k}$ in the case of the minimal sum of absolute deviations.

Step 5. Update the estimation of the matrix $P$ :

$$
P_{k}=\left[1-\chi v_{k}\right] P_{k-1} \rho^{-1}, \quad P_{k} \in \mathbb{R}^{m \times m} .
$$

Step 6. Calculate the vector $\Theta^{*}$ of the biased model coefficients (9) if it is necessary:

$$
\begin{aligned}
\Theta^{*}(k) & =\Theta^{0}(k)-\xi\left[Q \Theta^{0}-q\right], \\
\xi & =P_{k} Q^{T}\left[Q P_{k} Q^{T}\right]^{-1},
\end{aligned}
$$

where $Q$ and $q$ are defined by the bias condition (5), e.g., like in Eqns. (22), (24) or (25), 


\section{Example of application}

The proposed approach was used for estimation of the model of dynamics of the steam conditioner in a power station (Fig. 1). The dynamics of steam conditioner injectors of II stage were investigated.

The basic function of the investigated water injector was to maintain steam temperature on a demanded level. The steam flow was defined by the actual load of the turbine and was kept constant. The control of temperature was simple-electrical controlled valves $v$ delivered water to a steam. Water was evaporating and reduced the temperature of the steam at the output. The control was fast and effective in reduction of disturbances: variable input steam temperature $T_{i}$ and steam humidity $\eta$. The signals $T_{i}, \eta, v$-valve position (percentage of closing) and $T_{o}$ - output temperature, were measured and available in control system. The data were collected at the sampling rate $\Delta=1 \mathrm{~s}$ and the quality of measurements for both temperatures $T_{i}$ and $T_{o}$ was equal to $0.1^{\circ} \mathrm{C}$ : for the humidity transients a transducer of 12-bit resolution was used. The control valve signal was a variable with a dead zone of $1 \%$ and the corresponding transducer was quite precise and had 16-bit resolution.

The control problem and the investigated models were restricted to a defined operating point - the defined load of the power system. Limited operation conditions

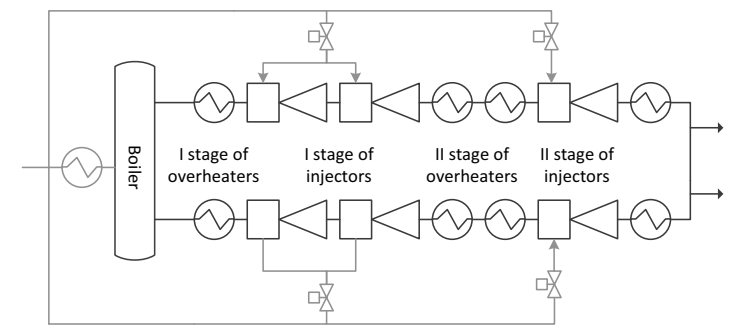

Fig. 1. Scheme of the steam-water flow in a power system.

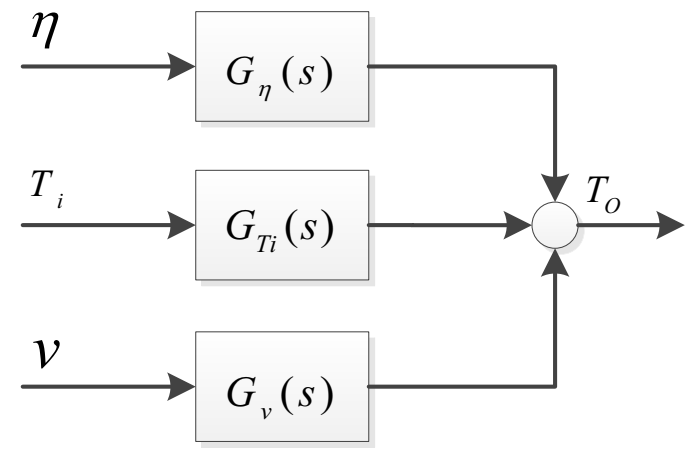

Fig. 2. Basic scheme of the investigated process dynamics.
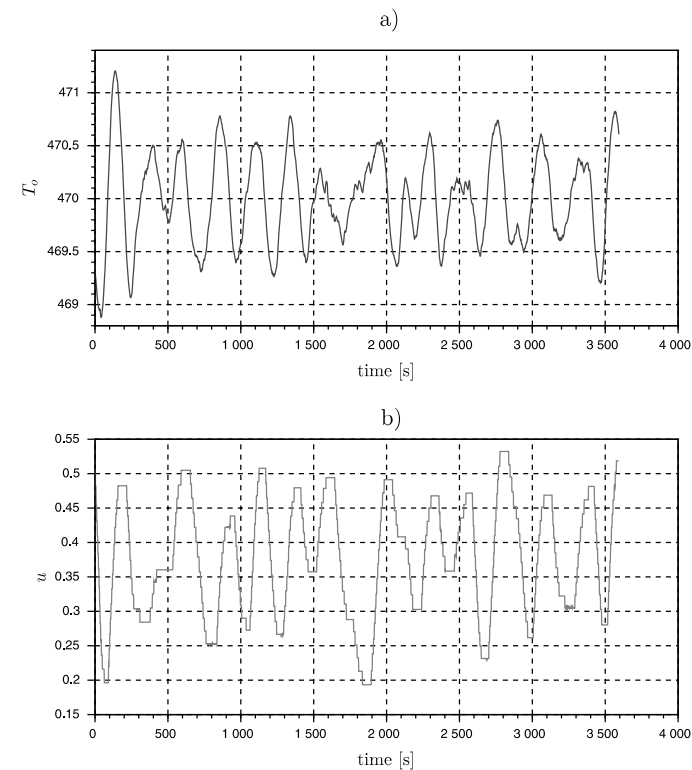

Fig. 3. Data used for identification: output temperature (a), valve action (b).

suggested investigation of linear transfer function models with a structure shown in Fig. 2.

The balance of steam enthalpy showed that more than $92-96 \%$ of output steam energy was delivered by the enthalpy of the input steam flow, and hence the transfer function $G_{T o T i}(s)$ will present a static gain of about 0.90 up to 0.91 with respect to the temperature of the input steam, reduced by injection of water that evaporated in the hot steam flow. The data used for identification were recorded during normal operation of the plant, subjected to a closed-loop control that has maintained a constant value of output steam temperature $T_{o}$, approximately $470^{\circ} \mathrm{C}$ (Fig. 3 (a)). This value was held by the injector valve position with the range [0.19,0.53] (1.0: closed, 0.0: open) (Fig. 3 (b)). The model of dynamics was investigated in the structure corresponding to Fig. 2. Statistical estimation methods were based on the performance index (2) in the form of the expected value of one-step prediction error variance, with the algorithm presented in Section 4 without bias (4).

The evaluation index (4) of the calculated models was based on the measure of the error in the form $e_{D}$,

$$
\begin{aligned}
e_{D}(k)= & T_{o}(k)-v(k) \hat{\Theta}, \\
v(k)= & {\left[\hat{T}_{o}(k-1), \ldots, \eta\left(k-1-d_{\eta}\right), \ldots,\right.} \\
& \left.\tau_{i}\left(k-1-d_{\tau}\right), \ldots, v\left(k-1-d_{v}\right), \ldots\right],
\end{aligned}
$$

where $\hat{T}_{o}(k-i)$ denotes the value of the output steam temperature estimated (not directly measured) by the model. As for synchronization of both the sequences, the estimated values $\hat{T}_{o}(k-i)$ have been replaced by the measured $T_{o}(k-i)$ values at first $n+d_{\max }$ (model order and maximal delay) time instants. Such an approach 


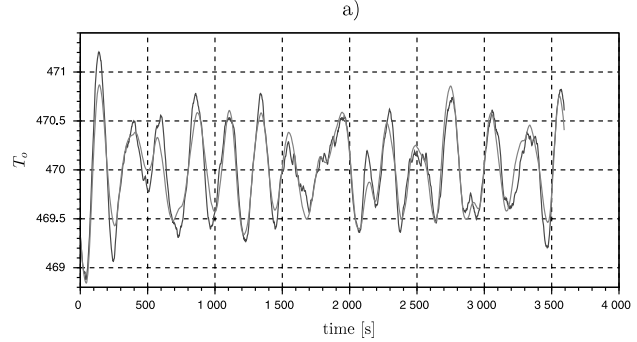

b)

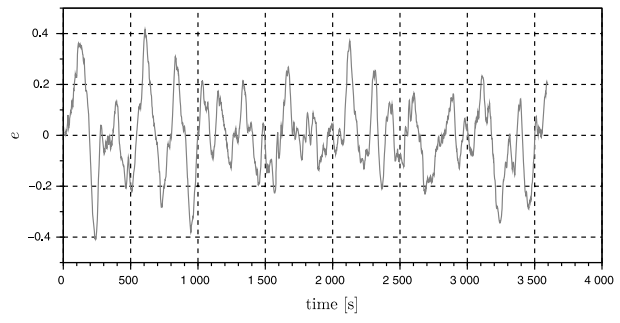

Fig. 4. Best linear model for $T_{o}$ fitted in terms of (4): plots of measured (dark grey) and estimated (light grey) $T_{o}$ (a), absolute error between $T_{o}$ and $\hat{T}_{o}$ (b).

should reveal real impact of each input $T_{i}, \eta$ and $v$ on estimation of the evolution of the output temperature $T_{o}$.

An example of identification and model evaluation is shown in Fig. 4 The best order, fitted in the class of linear, three input (humidity $\eta$, valve $v$, inlet temperature $T_{i}$ ) models, resulted in quite good effects of modeling in terms of (4) (see Fig. 4). The optimal model structure was as follows: order $n=9$, delays in process inputs $d_{\eta}=5$, $d_{v}=5$ and $d_{T_{i}}=0$ at the sampling interval $\Delta=1 \mathrm{~s}$. The obtained transfer functions can be easily reduced to forms of order four or five. In Fig. 4(a) a direct comparison of the measured output $T_{o}$ and the one estimated on the basis of the model is shown, and in Fig. 4(b) the error between both the signals is shown.

The mean error value was less than about $0.024 \%$, which corresponded to the mean error value equal to $0.126^{\circ} \mathrm{C}$, but the analysis of the resulting model structure has created some confusion. The static gain factor for the humidity impact $\eta$ was -11.52 , for the closing of the water inlet valve $v$ it was 1.339 , and for the input steam temperature $T_{i}$ it was 0.475 . The last value was too small and the static gain for valve impact was estimated by the process operator as rather too small. It was clear that conditions for efficient identification were violated, but the possibility of conducting an active experiment on a normally working turbine system was limited. After determination of the optimal (in terms of the performance index (4)) discrete time transfer functions for the discussed inputs $(\eta, v$, $T_{i}$ ) of order $n=9$, reduction in these functions combined with transformation into continuous time domain was performed. This transformation was based on minimization of the distance between the frequency

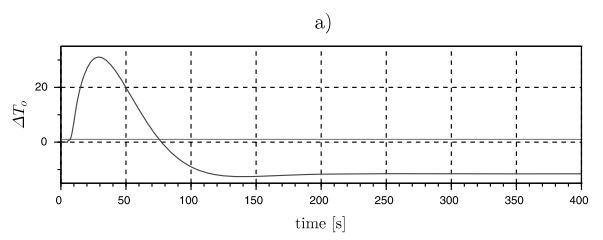

b)
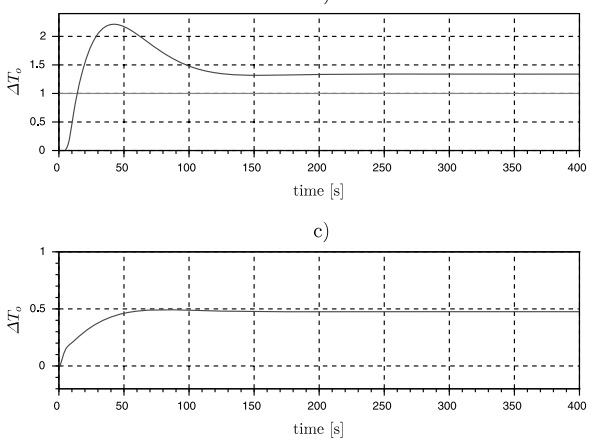

Fig. 5. Estimated step responses of $T_{o}$ with respect to $\eta$ (a), $v$ (b), $T_{i}$ (c) for the optimal model.

characteristic determined for the original transfer function and the characteristic of the continuous time transfer function of lower order preceded by the $\mathrm{ZOH}$ element (Janiszowski, 2014). The reduced transfer functions for variations in the output $\Delta T_{o}$ and variations in the investigated inputs $\Delta \eta, \Delta v, \Delta T_{i}$ were equal to

$$
\begin{aligned}
G_{\Delta T_{o} \Delta \eta}(s) & \\
= & \frac{-11.55+1815 s-1537.5 s^{2}+1497.2 s^{3}}{1+36.319 s+573.9 s^{2}+847.25 s^{3}+915.75 s^{4}} \\
& \times e^{-5 s}
\end{aligned}
$$

$$
\begin{aligned}
& G_{\Delta T_{o} \Delta v}(s) \\
& =\frac{1.339+85.592 s-12.233 s^{2}+58.516 s^{3}}{1+36.402 s+577.06 s^{2}+896.84 s^{3}+852.51 s^{4}} \\
& \quad \times e^{-5 s}, \\
& G_{\Delta T_{o} \Delta T_{i}}(s) \\
& =\frac{0.4754+10.293 s+54.555 s^{2}-16.543 s^{3}}{1+36.957 s+595.78 s^{2}+1134.53 s^{3}+1324.15 s^{4}} .
\end{aligned}
$$

Figure 5 presents the step responses of this model. The humidity impact, as observed in Fig. 5 a), is very dynamic; however, in steady state it represents a small effect. The change in the humidity $\eta$ should involve a similar variation in $T_{o}$, due to the operator experience, but the effect of such strong non-minimum phase behavior of the valve was not directly observed.

For estimation of a more realistic injector model, the static gain of the inlet steam temperature $T_{i}$ was fixed at 0.91 and the algorithm presented in Section 4 was used. 


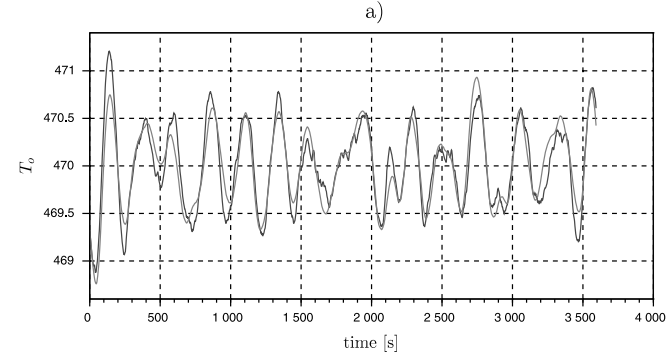

b)

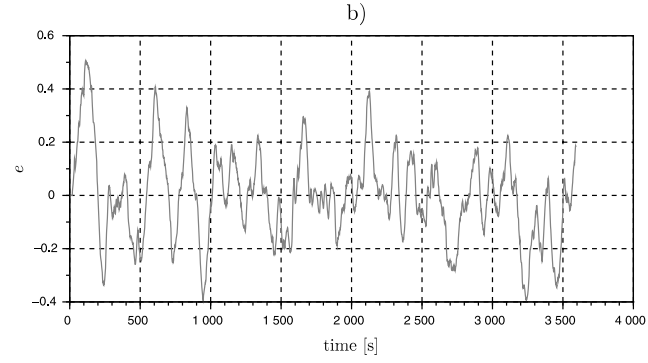

Fig. 6. Biased linear model for $T_{o}$ fitted in terms of (4) for the fixed static gain of $T_{i}$ : plots of $T_{o}$ (dark grey) and $\hat{T}_{o}$ (light grey) (a), absolute error between $T_{o}$ and $\hat{T}_{o}$ (b).

The obtained best model for the injector installation was of the following structure: order $n=14$, delays at inputs $d_{\eta}=5, d_{v}=5$ and $d_{T_{i}}=0$ with the mean value of the error (modeled as in the previous case) equal to $0.166^{\circ} \mathrm{C}$. For the order $n=9$ and the same delays, the error was estimated at $0.169^{\circ} \mathrm{C}$, and hence a more compact model is discussed here. The gains were estimated for $\eta=-8.834$, the water inlet valve $v=2.83$ and the input steam temperature $T_{i}=0.91$. The results of modeling in the sense of (4) are presented in Fig. 6 After reduction of the transfer functions of the investigated models to the fourth order, and approximation to the continuous time domain, the corresponding representations were obtained:

$$
\begin{aligned}
& G_{\Delta T_{o} \Delta \eta}(s) \\
& =\frac{-8.834+1625.9 s-2439.3 s^{2}+1580.73 s^{3}}{1+44.155 s+663.136 s^{2}+977.99 s^{3}+1074.6 s^{4}} \\
& \quad \times e^{-5 s}, \\
& G_{\Delta T_{o} \Delta v}(s) \\
& =\frac{2.836+60.338 s-64.036 s^{2}+48.823 s^{3}}{1+43.286 s+626.9 s^{2}+1072.4 s^{3}+1027.2 s^{4}} \\
& \quad \times e^{-5 s}, \\
& G_{\Delta T_{o} \Delta T_{i}}(s) \\
& =\frac{0.91+12.72 s+39.2 s^{2}+3.57 s^{3}}{1+44.9 s+695.94 s^{2}+1449.51 s^{3}+1746.4 s^{4}} .
\end{aligned}
$$

The impact of steam humidity showed again strong non-minimal-phase behavior. The step responses for the
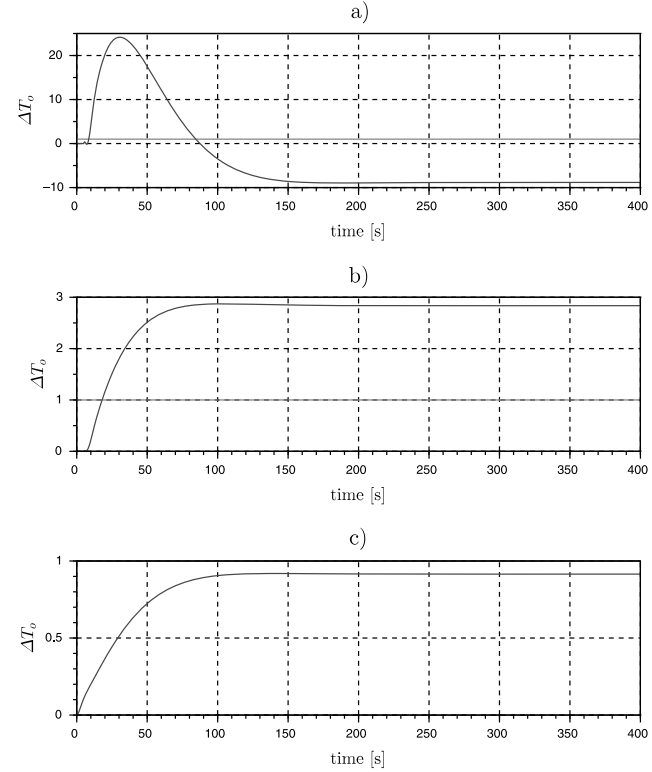

Fig. 7. Estimated step response of $T_{o}$ with respect to $\eta$ (a), $v$ (b), $T_{i}(\mathrm{c})$ for the biased model.

same inputs variations are presented in Fig. 7 The impact of input temperature $\Delta T_{i}$ represents the demanded level -0.91 . The error measure (Fig. 6 b)), was only slightly greater than the one observed in the case of the optimal model (Fig. 4b)). It should be mentioned that the impact of the unmeasured, but surely very important signal, i.e., the steam flow, may have influence on the model quality and the fitting of the optimal model.

\section{Conclusions}

The proposed approach of special biased estimation has been used in practice for determination of dynamics for process running in a closed control loop. This case is known as a difficult problem, especially in the presence of constant compensation of disturbance impacts. The "free" estimation may induce the effect of a loss of identifiability, hence one way is biased estimation, with some fixed a priori knowledge of the model features. As the presented results showed (Figs. 4 and 6), both the dynamic models are quite good in the sense of the performance index (4), but the latter was closer to the process operator's expectation and can be accepted by the end-user, i.e., a technology engineer. The proposed approach is applicable to the problem of known static gains, but it can be used for other problems, too, e.g., known time constants, the presence of integration, known impacts of two mutually compensating inputs, etc. In the given case of the steam conditioner, the problem of identification in the closed-loop system can be combined with the latter effect-it is well known that humidity of steam in front of the injector is strongly related to 
its temperature and therefore the above-mentioned effect may deteriorate identification quality.

The condition introduced by the relation (5) has the form of a linear bound of the linear model coefficients $\Theta$, which has to be satisfied by the estimated model. This condition can be relaxed by introduction of a weaker one. The model performance index can be a convex penalty function defined on the estimated model property $\kappa_{0}$ defined by the distance measure $\rho\left|\kappa-\kappa_{0}\right|$, where the $\kappa$ is a property expected in the estimated model. Then the goal of estimation will be defined by the minimum of $I_{D}(\hat{\Theta})+\rho\left|\kappa-\kappa_{0}\right|$, where the model error measure will be combined with the distance from the above-mentioned property $\kappa$.

\section{References}

Aguire, L.A., Barroso, M.F.S., Saldanha R.R., and Mendes E.M.A.M. (2004). Imposing steady-state performance on identified nonlinear polynomial models by means of constrained parameter estimation, IEE Proceedings: Control Theory and Applications 151(2): 174-179.

Astrom, K.J. (1983). Theory and applications of adaptive control-a survey, Automatica 19(5): 471-486.

Bun, M.J.G. and Carree, M.A. (2000). Bias-corrected estimation in dynamic panel data models, Journal of Business and Economic Statistics 23(2): 200-210.

Draper, N.R. and Smith, H. (1998). Applied Regression Analysis, 3rd Edition, Wiley, Berlin.

Eykhoff, P. (1974). System Identification Parameter and State Estimation, John Wiley and Sons, London/New York, NY.

Ferretti, G., Maffezzoni, C. and Scattolini, R. (1991). Recursive estimation of time delay in sampled data systems, Automatica 27(4): 653-661.

Gautier, M. and Briot, S. (2011). New method for global identification of the joint drive gains of robots using a known payload mass, Proceedings of the IEEE Conference on Inteligent Robots and Systems, San Francisco, CA, USA, pp. 25-30.

Goodwin, C.G. and Welsh, J.S. (2002). Bias issues in closed loop identification with application to adaptive control, Communication in Information and Systems 2(4): 349-370.

Gourieroux, C., Phillips, P.C.B. and Yu, J. (2010). Indirect inference for dynamic panel models, Journal of Econometrics 157(1): 68-77.

Hayakawa, K. (2010). The effects of dynamic feedbacks of LS and MM estimator accuracy in panel data models; some additional results, Journal of Econometrics 159(1): 202-208.

Heath, W.P. (2001). Bias of indirect non-parametric transfer function for plants in closed loop, Automatica 37(10): 1529-1540.

Isermann, R. (1988). Identifikation dynamischer Systeme, Springer, Berlin
Janiszowski, K. (1998). Towards least sum of absolute errors estimation, IFAC Symposium on Large Scale Systems, LSS'98, Patras, Greece, pp. 613-619.

Janiszowski, K.B. (2014). Approximation of linear dynamic process model using the frequency approach and a non-quadratic measure of the model error, International Journal of Applied Mathematics and Computer Science 24(1): 99-111, DOI: 10.2478/amcs-2014-0008.

Kiviet, J.F. (1995). On bias, inconsistency and efficiency of various estimators in dynamic panel data models, Journal of Econometrics 68(1): 53-78.

Kowalczuk, Z. and Kozłowski, E. (2000). Continuous-time approaches to identification of continuous-time systems, Automatica 36(8): 1229-1236.

Kozłowski, E. and Kowalczuk, Z. (2007). Robust to measurement faults parameter estimation algorithms in problems of systems diagnostics, in Z. Kowalczuk and B. Wiszniewski (Eds.), Intelligent Information Extraction for Diagnostic Purposes, PWNT, Gdańsk, pp. 221-240.

Ljung, L. (1999). System Identification-Theory for the User, Prentice Hall, Englewood Cliffs, NJ.

Ljung, L. and Foorsell, U. (1998). Bias, variance and optimal experiment design: Some comments on closed loop identification, in D. Norman-Cyrot (Ed.), Perspectives in Control, Springer-Verlag, Berlin, pp. 205-216.

Ljung, L. and Gunnarson, S. (1990). Adaptation and tracking in system identification-a survey, Automatica 26(1): 7-21.

Ninness, B.M., Hjalmarson, H. and Gustafsson, F. (1999). The fundamental role of general othonormal bases in system identification, IEEE Transactions on Automatic Control 44(7): 1384-1406

Norton, J.P. (1980). An Introduction to Identification, Academic Press, London/ New York, NJ.

Phillips, P. and Sul, D. (2007). Bias in dynamic panel estimation with fixed effects, incidental trends and cross section dependence, Journal of Econometrics 137(1): 162-188.

Söderström, T., Fan, H., Carlsson, B. and Bigi, S. (1997). Least squares parameter estimation of continuous-time ARX models from discrete-time data, IEEE Transactions on Automatic Control 42(5): 659-673.

Söderström, T. and Stoica, P. (1989). System Identification, Prentice Hall, Hertfordshire.

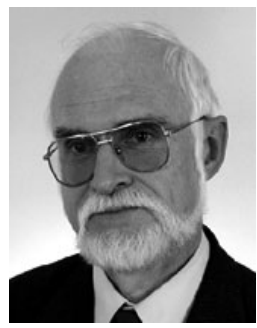

Krzysztof Janiszowski is the head of the Activators for Automation and Robotics Division of the Mechatronics Department of the Warsaw University of Technology, specializing in identification, modeling, and control techniques. His research areas include identification of industrial plants, algorithms for control and fast prototyping in fluidic servo-mechanisms, models of human circulation systems, software packages for modeling and control of industrial processes. 


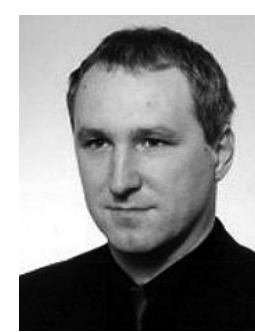

Pawel Wnuk is a lecturer at the Institute of Automatic Control and Robotics, Warsaw University of Technology. In his scientific research works he is mostly concerned with the modeling of system dynamics and the use of mass intelligence algorithms. His areas of interest include identification of industrial plants, models and diagnostics of technical and medical systems, software for supervision, diagnostics and telemetry.

Received: 19 June 2015

Revised: 7 December 2015

Re-revised: 22 April 2016

Accepted: 4 June 2016 